Pacific Journal of Mathematics

GAUSSIAN PROCESSES WITH STATIONARY INCREMENTS 


\title{
GAUSSIAN PROCESSES WITH STATIONARY INCREMENTS POSSESSING DISCONTINUOUS
} SAMPLE PATHS

\author{
Michael B. Marcus
}

In this paper a sufficient condition is given on the covariance of a stationary Gaussian process for it to have sample paths that are unbounded in all intervals. This result is an extension of a result of Belyaev.

It is also shown that an obvious analogue of this result applies to Gaussian processes with stationary increments.

Let $X(t)$ be a real valued Gaussian process with stationary increments such that $E\{X(t)\}=0$ and

$$
E\left\{(X(t+h)-X(t))^{2}\right\}=\sigma^{2}(h),
$$

$\sigma(h) \rightarrow 0$ as $h \rightarrow 0$. Suppose that $\sigma^{2}(h)$ is concave in $[0, \delta]$ for some $\delta>0$ and that

$$
\sigma^{2}(h) \geqq \frac{C}{|\log | h||}, \quad C>0,
$$

for $h \in[0, a]$, for some $a>0$. Then it follows that at any point $t_{\text {, }}$, with probability one, the sample paths of $X(t)$ are discontinuous. This result was obtained by Belyaev [1] for stationary Gaussian processes; its extension to Gaussian processes with stationary increments is trivial.

Suppose $X(t)$ is a real valued stationary Gaussian process, normalized so that $E\left\{(X(t))^{2}\right\}=1$. Denote its covariance by $\varphi(h)$; thus $\sigma^{2}(h)=2(1-\varphi(h))$. Let $\Phi$ be the class of all covariance functions $\varphi(h)$ such that $\varphi(0)=1, \varphi(h)$ is convex in some interval $[0, \delta], \delta>0$ ( $\delta$ need not be the same for all $\varphi \in \Phi)$, and $2(1-\varphi(h))$ satisfies Eq. (2) for $h \in[0, a]$, for some $a>0$. Then Belyaev's result asserts that the stationary Gaussian processes defined by the covariance functions in $\Phi$ have discontinuous sample paths. The main result of this paper is Theorem 2, which is equivalent to the following statement: Let $\Gamma$ be the set of all normalized covariance functions. Let $\Phi$ be as defined above. Then if $\gamma(h) \in \Gamma, \varphi(h) \in \Phi$, with probability one the stationary Gaussian process with covariance $\gamma(h) \cdot \varphi(h)$ has sample paths that are discontinuous in every interval. In other words, the class of stationary Gaussian processes with discontinuous sample paths is extended from $\Phi$ to $\Gamma \Phi$. A similar result is obtained for Gaussian processes with stationary increments.

Once it is shown that the sample paths of a stationary Gaussian 
process are discontinuous with probability greater than zero, Belyaev's result [1] - that either the sample paths of a stationary Gaussian process are continuous with probability one or else with probability one they are unbounded in every interval-further describes the behavior of the paths.

An unsolved question is the exact nature of the set of covariance functions $\Gamma \Phi$. The set contains $\Phi$ since $\Gamma$ contains the constant 1 . Also, $\Gamma \Phi$ is larger than $\Phi$, since we can take for $\gamma \in \Gamma$ a nowhere differentiable function (obtained from the theory of lacunary series). Therefore, a $\gamma \varphi$ can be found that is nowhere differentiable and hence not convex in some interval $[0, a], a>0$. This covariance function will not be contained in $\phi$. The problem of identifying the product of a characteristic function of a probability measure with all other characteristic functions has been posed in harmonic analysis, but not much seems to be known about it.

2. The first lemma is probabilistic: in the rest of the paper we are concerned with evaluating determinants of covariance matrices.

Lemma 1. Let $X_{1}, \cdots, X_{n}$ be linearly independent real valued Gaussian random variables with mean zero and finite variances. Let $D_{i}$ be the determinant of the $i^{\text {th }}$ principal minor of their covariance matrix $R$. Define $\rho_{i}=D_{i-1} / D_{i} i=1, \cdots, n$. $D_{0} \equiv 1$. Then

$$
P\left\{\max _{1 \leqq i \leqq n}\left|X_{i}\right| \leqq a\right\} \leqq \prod_{i=1}^{n} \sqrt{\frac{2}{\pi}} \int_{0}^{a\left(\rho_{i}\right)^{1 / 2}} e^{-\xi^{2} / 2} d \xi .
$$

Proof. We have

$$
\begin{aligned}
P\left\{\max _{1 \leqq i \leqq n}\left|X_{i}\right| \leqq a\right\} & \\
& =\frac{|A|^{1 / 2}}{(2 \pi)^{n / 2}} \int_{\left|x_{i}\right| \leqq a} \cdots \int \exp \left\{-\frac{1}{2} \sum_{i, j} A_{i j} x_{i} x_{j}\right\} d x_{i} \cdots d x_{n},
\end{aligned}
$$

where $A=R^{-1}$, and $|A|$ denotes the determinant of $A$.

Since the random variables are square integrable functions on an $n$-dimensional Gaussian measure space, they can be considered as elements in a Hilbert Space. Their covariance corresponds to the inner product. Using the Gram-Schmidt orthogonalization procedure, we can extract an orthonormal sequence $\xi_{i}=\sum_{j} P_{i j} X_{j}$, where $P^{T} P=A$. The functions $\xi_{i}$ have the following form:

$$
\xi_{\imath}=\left(\rho_{i}\right)^{1 / 2} X_{i}+f_{i}\left(\xi_{i-1}, \cdots, \xi_{1}\right), \quad i=1, \cdots, n
$$

(See [4], p. 37). The change of variables $\xi_{i}=\sum_{j} P_{i j} X_{j}$ diagonalizes the 
quadratic form in Eq. (3) and the integral is equal to

$$
\prod_{i=1}^{n} \frac{1}{\sqrt{2 \pi}} \int_{B_{i}} \exp \left\{-\xi_{i}^{2} / 2\right\} d \xi_{i} .
$$

The interval of integration $B_{i}$ is given by the inequality

$$
-\left(\rho_{i}\right)^{1 / 2} a+f\left(\xi_{i-1}, \cdots, \xi_{1}\right) \leqq \xi_{i} \leqq\left(\rho_{i}\right)^{1 / 2} a+f_{i}\left(\xi_{i-1}, \cdots, \xi_{1}\right) .
$$

Because the density function of the Gaussian distribution is symmetric and decreases monotonically as $\left|\xi_{i}\right|$ increases, the integrals in Eq. (4) are increased if we take $\left|\xi_{i}\right| \leqq\left(\rho_{i}\right)^{1 / 2} a$. This observation proves the lemma.

Using Ostrowski's results [3] on matrices with dominant principal diagonals, an upper bound for $D_{i-1} / D_{i}$ can be obtained when the determinants $D_{i}$ are those of a covariance matrix formed by the increments of a stationary Gaussian process which itself has a covariance that is convex in $[0, \delta]$ for some $\delta>0$. For the sake of completeness of this paper, Ostrowski's result will be restated.

Let $A=\left\{a_{\mu_{\nu}}\right\}$ be a real valued matrix with positive diagonal elements such that the sum of the absolute values of all the off diagonal elements in a given row is less than the diagonal element in that row. Such a matrix $A$ is called a matrix with dominant principal diagonal. For these matrices we define $\alpha_{\mu_{\nu}}=\left|a_{\mu^{\prime \prime}}\right|, \mu, \nu=1, \cdots, n$ and

$$
s_{\mu^{\prime}}=\sum_{\nu=1}^{n} \alpha_{\mu^{\nu}}-\alpha_{\mu^{\mu}}=\sigma_{\mu} \alpha_{\mu^{\mu}}, \quad 1 \leqq \mu, \nu \leqq n .
$$

Note that $\sigma_{\mu}<1$. The following lower bound is obtained for $|A|$ :

$$
|A| \geqq \prod_{\mu=1}^{n}\left(\alpha_{\mu / \mu}-s_{\mu}\right) \text {. }
$$

Equation (6) shows that a matrix with dominant principle diagonal is strictly positive definite.

Let $D_{i}$ be the determinant of $\left\{a_{\mu_{2}}\right\} \mu, \nu=1, \cdots, i$. In a manner similar to Eq. (5), we define

$$
s_{\mu}^{i}=\sum_{\nu=1}^{i} \alpha_{t^{\nu}}-\alpha_{\mu^{\prime \prime}}=\sigma_{\mu^{\prime}}^{2} \alpha_{\mu^{\prime \prime}}, \quad 1 \leqq \mu \leqq i,
$$

and

$$
t_{\imath}^{i}=\max \sigma_{k}^{i}, \quad(k=1, \cdots, i-1) .
$$

Then it follows that

$$
\left(\alpha_{i i}+t_{i}^{i} s_{i}^{i}\right) D_{i-1} \geqq D_{i} \geqq\left(\alpha_{i i}-t_{\imath}^{i} s_{i}^{i}\right) D_{i-1} .
$$

Note that $s_{\imath}^{i}=\sum_{\substack{\nu=1 \\ i-1}} \alpha_{i}$ is the sum of the absolute values of the elements 
in the $i^{\text {th }}$ row of $A$ that lie to the left of the diagonal element.

We shall now exhibit a collection of Gaussian random variables which have as their covariance matrix a matrix with dominant principal diagonal. Let $\gamma(h)$ be the covariance of a stationary Gaussian process and suppose that $\gamma(h)$ is convex for $h \in[0, \delta]$ for some $\delta>0$, and that $\gamma(\delta-\varepsilon)>\gamma(\delta)>0(0<\varepsilon \leqq \delta)$. Let $0<t_{1}<t<t_{n} \leqq \delta$ be a partition of $[0, \delta]$. The covariance matrix of $\left(X\left(t_{j}\right)-X\left(t_{j-1}\right)\right), j=1, \cdots, n$ is a matrix with dominant principal diagonal. This is easy to verify since all of the off diagonal terms of the $n$-dimensional covariance matrix of $X\left(t_{j}\right)-X\left(t_{j-1}\right), j=1, \cdots, n$ are negative due to the convexity of $\gamma(h)$. Thus the absolute values of the off diagonal elements in a given row can be added. In the case under consideration, $s_{\imath}^{i} \leqq \alpha_{i i} / 2=$ $\sigma^{2}\left(t_{i}-t_{i-1}\right) / 2$. Since $t_{i}^{i} \leqq 1$ we have

$$
\frac{D_{i-1}}{D_{i}} \leqq \frac{2}{\sigma^{2}\left(t_{i}-t_{i-1}\right)}
$$

Also note that the functions $D_{i-1} / D_{i}$ for the covariance matrices of the random variables $X\left(t_{j}\right)-X\left(t_{j-1}\right), j=1, \cdots, i$ and the functions $D_{i-1} / D_{i}$ for the covariance matrices of the random variables $X\left(t_{j}\right)-X\left(t_{0}\right)$, $j=1, \cdots, i$ are equal. This follows from row and column operations on the matrices. Referring to Lemma 1 and using Eq. (8), the following lemma is obtained.

Lemma 2. Let $X(t)$ be a real valued stationary Gaussian process with covariance $\gamma(h)$ which is convex for $h \in[0, \delta]$ for some $\delta>0$. Let $t_{0}<t_{1}<\cdots<t_{n},\left(t_{n}-t_{0}\right) \leqq \delta$. Then

$$
\operatorname{Prob}\left\{\max _{1 \leqq i \leqq n}\left|\xi_{i}\right| \leqq a\right\} \leqq \prod_{i=1}^{n} \sqrt{2 / \pi} \int_{0}^{\sqrt{2} a / \alpha_{i i}} e^{-x^{2} / 2} d x,
$$

where $\xi_{i}$ can be either $X\left(t_{i}\right)-X\left(t_{i-1}\right)$ or $X\left(t_{i}\right)-X\left(t_{0}\right)$ and $\alpha_{i i}=$ $\sigma\left(t_{i}-t_{i-1}\right), i=1, \cdots, n$.

Using Lemma 2, the following theorem is easily obtained.

THEOREM 1. Let $X(t)$ be a real valued stationary Gaussian process with covariance function $\gamma(h)$ that is convex for $h \in[0, \delta]$ for some $\delta>0$. Let $\sigma(h)=2(1-\gamma(h))$. Then if

$$
\sigma(h) \geqq \frac{C}{|\log | h||}, \quad C>0,
$$

$h \in[0, a]$, for some $a>0$, it follows that almost all sample functions of $X(t)$ are unbounded in every interval.

The result in Theorem 1 was first obtained by Belyaev, who 
employed a lemma due to Ventcel which is similar to Lemma 2. An alternate way of proving Theorem 1 is to use the Chung-Erdos version of the Borel-Cantelli lemma [2]. Theorem 1 is contained in Theorem 2. It has been displayed separately because its proof is simple and because it is used to conclude the proof of Theorem 2 .

In preparation for Theorem 2, we shall prove some lemmas on determinants associated with Toeplitz matrices. A matrix $\left\{c_{j, k}\right\} j, k=0$, $1, \cdots, n$ is a Toeplitz matrix if it is positive definite and if $c_{j, k}=c_{j-k}$. We shall normalize these numbers by taking $c_{0}=1$. The elements of a Toeplitz matrix can be represented as the Fourier-Stieltjes transforms of a distribution function $\alpha(x)$ on the unit circle,

$$
c_{k}=\frac{1}{2 \pi} \int_{-\pi}^{\pi} e^{-i k x} d \alpha(x) .
$$

Conversely, distribution functions on the unit circle give rise to positive definite sequences $\left\{c_{k}\right\}$ such that finite subsets of these sequences determine Toeplitz matrices. If the distribution function has at least $n+1$ points of increase, the determinants of the matrices $\left\{c_{j-k}\right\}, j$, $k=0,1, \cdots, i, 0<i \leqq n$ are strictly positive. A distribution function will be said to be of order $k$ if it has at least $k$ points of increase.

Let $\left\{c_{k}\right\} k=0, \pm 1, \cdots$, be a positive definite sequence and $\alpha(x)$ the corresponding distribution function. We shall denote the determinant of the matrix given by $c_{0}, c_{-1}, c_{1}, \cdots, c_{-n}$ by $D_{n+1}(\alpha)$. Also, the function $D_{n}(\alpha) / D_{n+1}(\alpha)$ will be referred to as $D_{n} / D_{n+1}(\alpha)$.

The following lemma is a minor modification of Theorem (a) on page 38 in Ref. 4.

Lemma 3. Let $\alpha(x)$ be a distribution function on $[-\pi, \pi]$ of order $n+1$ and $g(z)$ be any polynomial of the $n^{\text {th }}$ degree in $e^{i x}$, $a_{0} z^{n}+a_{1} z^{i n-1}+\cdots+a_{n}, z=e^{i x}$, subject to the condition that $\left|a_{0}\right|=1$. Then

$$
\frac{D_{n+1}}{D_{n}}(\alpha)=\min _{g(z)} \frac{1}{2 \pi} \int_{-\pi}^{\pi}|g(z)|^{2} d \alpha(x),
$$

where the minimum is taken over all the polynomials $g(z)$.

Since $\alpha(x)$ defines a probability measure on the unit circle, so does its translate $\alpha(x-\xi)$. Define this new distribution function by $\alpha_{\xi}(x)$. The proof of the following lemma follows immediately from Lemma 3.

LEMMA 4. Let $\alpha(x)$ be a distribution function of order $n+1$ on $[-\pi, \pi]$. Then 


$$
\frac{D_{n+1}}{D_{n}}(\alpha(x))=\frac{D_{n+1}}{D_{n}}\left(\alpha_{\xi}(x)\right) \text {. }
$$

Let $\Theta$ be the set of all probability measures on the unit circle. We shall now consider the set of probability measures $\{\alpha * \theta, \theta \in \Theta\}$, where $*$ denotes convolution.

Lemma 5. Let $\alpha(x)$ be a distribution of order $n+1$ on $[-\pi, \pi]$, and let $\theta$ be any distribution function on $[-\pi, \pi]$. Then

$$
\frac{D_{n+1}}{D_{n}}(\alpha) \leqq \frac{D_{n+1}}{D_{n}}(\alpha * \theta)
$$

Proof. Let $\theta_{\xi},(-\pi \leqq \xi \leqq \pi)$ be the extreme points of $\Theta$, i.e., the measures that assign probability one at the point $\xi$. Clearly the extreme points of $\{\alpha * \theta, \theta \in \theta\}$ are contained in the extreme points of $\left\{\alpha * \theta_{\xi}\right\}$. Consider the convolution of $\alpha$ with an element in the convex hull of $\left\{\theta_{\xi}\right\}$, i.e., $\alpha * \sum_{\jmath=1}^{n} t_{j} \theta_{\xi j}, \sum_{\jmath=1}^{n} t_{j}=1$. Then

$$
\frac{D_{n+1}}{D_{n}}(\alpha)=\sum_{j=1}^{n} t_{j} \frac{D_{n+1}}{D_{n}}\left(\alpha * \theta_{\xi j}\right) \leqq \frac{D_{n+1}}{D_{n}}\left(\alpha * \sum_{j=1}^{n} t_{j} \theta_{\xi j}\right) .
$$

The equality in Eq. (12) follows by Lemma 4, the inequality follows from Eq. (11).

Since $D_{n+1} / D_{n}$ is a uniformly continuous function of the appropriate $2 n+1$ Fourier-Stieltjes coefficients, Eq. (12) can be extended to the weak closure of the convex hull of $\left\{\alpha * \theta_{\xi}\right\}$. This is $\{\alpha * \theta, \theta \in \Theta\}$ itself. Thus, the lemma is proved.

Let $X(t)$ be a normalized stationary Gaussian process with covariance $\varphi$. Consider the stationary sequence $\{\varphi(j h)\}, j=0, \pm 1, \cdots$, and let $\alpha(x)$ be the probability distribution on the unit circle associated with $\{\varphi(j h)\}$. For this same process $X(t)$, consider the stationary sequence of Gaussian random variables $\{X(t+j h)-X(t+(j-1) h)\} j=0$, $\pm 1, \cdots$. Denote the elements of the covariance matrix of these random variables by $d_{n}$, i.e.,

$$
\begin{aligned}
d_{n} & =E\{(X(t+j h)-X(t+(j-1) h)(X(t+k h)-X(t+(k-1) h)\} \\
& =2 \varphi((j-k) h)-\varphi((j-k-1) h)-\varphi((j-k+1) h)
\end{aligned}
$$

where $n=j-k$. Thus, we have the following equation for $d_{n}$,

$$
d_{n}=\frac{1}{2 \pi} \int_{-\pi}^{\pi} e^{-i n x}\left|e^{i x}-1\right|^{2} d \alpha(x)
$$


Also, $d_{n}$ is a positive definite sequence; it is defined by the distribution function $\alpha_{2}(x)=\int_{-\pi}^{x}\left|e^{i y}-1\right|^{2} d \alpha(y)$. We shall normalize the sequence $d_{n}$ by dividing it by $d_{0}$; thus an alternate representation for Eq. (13) is

$$
d_{n} / d_{0}=\frac{1}{2 \pi} \int_{-\pi}^{\pi} e^{-i n x} d\left(\alpha_{2}(x) / d_{0}\right)
$$

LEMmA 6. Let $\alpha(x)$ be a distribution function of order $n+2$ and let $\alpha_{2}(x)$ be the distribution function corresponding to the second difference of the coefficients of $\alpha(x)$ (i.e., $c_{n}$ and $d_{n}$ as given above). Then

$$
\frac{D_{n+2}}{D_{n+1}}(\alpha(x)) \leqq \frac{D_{n+1}}{D_{n}}\left(\alpha_{2}(x)\right)
$$

Proof.

$$
\frac{D_{n+1}}{D_{n}}\left(\alpha_{2}(x) / d_{0}\right)=\min _{g(z)} \frac{1}{2 \pi} \int_{-\pi}^{\pi}|g(z)|^{2}\left|e^{i x}-1\right|^{2} d\left(\alpha(x) / d_{0}\right) .
$$

Note that the $1 / d_{0}$ factor can be cancelled from each side. The polynomial $g(z)=a_{0} z^{n}+a_{1} z^{n-1}+\cdots+a_{n}$ is subject to the restriction that $\left|a_{0}\right|=1$. Equation (14) can also be written as

$$
\frac{D_{n+1}}{D_{n}}\left(\alpha_{2}(x)\right)=\min _{f(z)} \frac{1}{2 \pi} \int_{-\pi}^{\pi}|f(z)|^{2} d \alpha(x),
$$

where the polynomial $f(z)=\alpha_{0} z^{n+1}+\alpha_{1} z^{n}+\cdots+\alpha_{n+1}$ is subject to the restrictions that $\left|\alpha_{0}\right|=1$ and that $\alpha_{n+1}=\alpha_{n}+\alpha_{n-1}+\cdots+\alpha_{0}$. The lemma follows directly from Eq. (15), since the right-hand side of Eq. (15) is greater than $D_{n+2} / D_{n+1}(\alpha(x))$. We can now prove Theorem 2.

THEOREM 2. Let $X(t)$ be a normalized stationary Gaussian process with covariance $\varphi(h)$ that is convex for $h \in[0, \delta]$, for some $\delta>0$. Suppose that $E\left\{(X(t+h)-X(t))^{2}\right\}=2(1-\varphi(h))=\sigma^{2}(h)$ satisfies the following inequality:

$$
\sigma^{2}(h) \geqq \frac{C}{|\log | h||}, \quad C>0,
$$

for $h \in[0, a]$, for some $a>0$. Then with probability one the sample paths of $X(t)$ are unbounded in every interval. Moreover, let $\Gamma$ be the set of all normalized covariance functions. If $\gamma(h) \in \Gamma$, then the stationary Gaussian process with covariance $\gamma(h) \cdot \varphi(h)$ has sample 
paths that are unbounded in every interval.

Proof. Without loss of generality we can assume $\delta \leqq a$ and that $\varphi(\delta-\varepsilon)>\varphi(\delta)>0$ for sufficiently small $\varepsilon>0$. Consider the sequence $\varphi\{[(j / 2 n) \delta]\} j=0,1, \cdots$. Because the Gaussian process is real, $\{\varphi(j / 2 n) \delta\}=\{\varphi(-j / 2 n) \delta\}$. Let $\alpha(x)$ be the distribution function defined by this sequence. By Eq. (6) we see that $\alpha(x)$ is of order $2 n+1$. Let $\mu(x)$ be the distribution function given by $\{\gamma(j / 2 n) \delta\} j=$ $0,1, \cdots$. Then $(\alpha * \mu)$ and $(\alpha * \mu)_{2}$ are of order $2 n$.

Let $X(t)$ be the stationary Gaussian process with covariance $\gamma(h) \cdot \varphi(h)$. Consider the random variables

$$
\left\{X\left(\frac{j}{2 n} \delta\right)-X\left(\frac{j-1}{2 n} \delta\right)\right\} j=1, \cdots, n+1 .
$$

They are linearly independent because $\left(\alpha^{*} \mu\right)_{2}$ is of order $2 n$. From Lemmas 6 and 5 we obtain

$$
\frac{D_{n}}{D_{n+1}}\left((\alpha * \mu)_{2}\right) \leqq \frac{D_{n+1}}{D_{n+2}}(\alpha * \mu) \leqq \frac{D_{n+1}}{D_{n+2}}(\alpha) \leqq \frac{2}{\sigma^{2}(\delta / 2 n)},
$$

where the last inequality is obtained using Ostrowski's results as follows: The term $D_{n+1} / D_{n 2}(\alpha)$ is a function on the Toeplitz matrix which has as its elements $\varphi\{[(j / 2 n) \delta]\} j=0,1, \cdots, n+1$. Perform the following row and column operations on this matrix: Subtract the $j^{\text {th }}$ row from the $j+1^{\text {st }}$ row $j=1,2, \cdots, n$, leaving the first row fixed, then subtract the $j^{\text {th }}$ column from the $j+1^{\text {st }}$ column $j=1,2, \cdots, n$, leaving the first column fixed. These operations leave $D_{n+1}(\alpha)$ and $D_{n+2}(\alpha)$ unchanged. The convexity of $\varphi(h)$ is used to show that all of diagonal terms of the resulting matrix are negative, and that the matrix has a dominant principle diagonal. The function $D_{n+1} / D_{n+2}(\alpha)$ is evaluated using Eq. (7). This accounts for the final inequality in Eq. (17).

The remainder of the proof follows from Lemma 2 and Theorem 1.

A result similar to Theorem 2 can be obtained for Gaussian processes with stationary increments. Theorem 2 extends the class of discontinuous stationary Gaussian processes from those for which

$$
E\left\{(X(t+h)-X(t))^{2}\right\}=2(1-\varphi(h))
$$

to those for which $E\left\{(X(t+h)-X(t))^{2}\right\}=2(1-\gamma(h) \cdot \varphi(h))$. The class of Gaussian processes with stationary increments that have discontinuous sample paths can also be extended from those processes for which $E\left\{(X(t+h)-X(t))^{2}\right\}=2(1-\varphi(h))$ for $h \in[0, \delta]$ for some $\delta>0$ and $\varphi \in \Phi$ to those processes for which 


$$
E\left\{(X(t+h)-X(t))^{2}\right\}=2(1-\gamma(h) \cdot \varphi(h))
$$

for $h \in[0, \delta]$ for some $\delta>0$, where $\gamma(h)$ is any normalized covariance function. The proof of this result for Gaussian processes with stationary increments is contained in the proof of Theorem 2, since in Theorem 2 we are concerned with the covariance matrix of the increments of a stationary Gaussian process. This matrix is the same as the covariance matrix of the increments of a Gaussian process with stationary increments as long as the function $\sigma^{2}(h)$ (i.e., as in Eq. (1)) is the same in both cases.

\section{REFERENCES}

1. Yu. K. Belyaev, Continuity and Hölder's conditions for sample functions of stationary Gaussian processes, Fourth Berkeley Symposium, Vol. 2, 1961.

2. M. B. Marcus, Hölder conditions for Gaussian processes with stationary increments, Trans. Amer. Math. Soc. October, 1968.

3. A. M. Ostrowski, Bounds for Determinants, Proc. Amer. Math. Soc. 3 (1952), 2630 .

4. G. Szego, and V. Grenander, Toeplitz Forms and Their Applications, University of California Press, 1958.

Received December 7, 1966.

RAND CORPORATION

NORTHWESTERN UNIVERSITY 



\section{PACIFIC JOURNAL OF MATHEMATICS}

\section{EDITORS}

\author{
H. ROYDEN \\ Stanford University \\ Stanford, California \\ R. R. Phelps \\ University of Washington \\ Seattle, Washington 98105
}

J. DUGUNDJI

Department of Mathematics

University of Southern California

Los Angeles, California 90007

RICHARD ARENS

University of California

Los Angeles, California 90024

\section{ASSOCIATE EDITORS}
E. F. BECKENBACH
B. H. NeumanN
F. WOLF
K. YosidA

\section{SUPPORTING INSTITUTIONS}

UNIVERSITY OF BRITISH COLUMBIA

CALIFORNIA INSTITUTE OF TECHNOLOGY

UNIVERSITY OF CALIFORNIA

MONTANA STATE UNIVERSITY

UNIVERSITY OF NEVADA

NEW MEXICO STATE UNIVERSITY

OREGON STATE UNIVERSITY

UNIVERSITY OF OREGON

OSAKA UNIVERSITY

UNIVERSITY OF SOUTHERN CALIFORNIA
STANFORD UNIVERSITY

UNIVERSITY OF TOKYO

UNIVERSITY OF UTAH

WASHINGTON STATE UNIVERSITY

UNIVERSITY OF WASHINGTON

AMERICAN MATHEMATICAL SOCIETY CHEVRON RESEARCH CORPORATION TRW SYSTEMS

NAVAL WEAPONS CENTER

Printed in Japan by International Academic Printing Co., Ltd., Tokyo, Japan 


\section{Pacific Journal of Mathematics}

\section{Vol. 26, No. $1 \quad$ November, 1968}

Efraim Pacillas Armendariz, Closure properties in radical theory......... 1

Friedrich-Wilhelm Bauer, Postnikov-decompositions of functors .......... 9

Thomas $\mathrm{Ru}-$ Wen Chow, The equivalence of group invariant positive definite

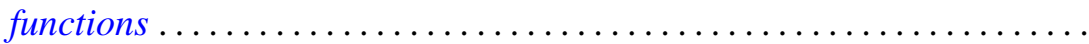

Thomas Allan Cootz, A maximum principle and geometric properties of

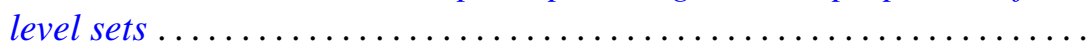

Rodolfo DeSapio, Almost diffeomorphisms of manifolds ............ 47

R. L. Duncan, Some continuity properties of the Schnirelmann density......

Ralph Jasper Faudree, Jr., Automorphism groups of finite subgroups of

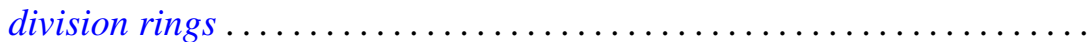

Thomas Alastair Gillespie, An invariant subspace theorem of $J$.

Feldman.........................................

George Isaac Glauberman and John Griggs Thompson, Weakly closed direct factors of Sylow subgroups .............................

Hiroshi Haruki, On inequalities generalizing a Pythagorean functional equation and Jensen's functional equation .....................

David Wilson Henderson, D-dimension. I. A new transfinite dimension.....

David Wilson Henderson, D-dimension. II. Separable spaces and

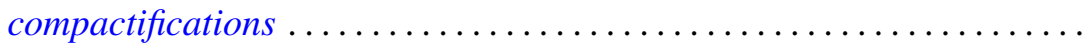

Julien O. Hennefeld, A note on the Arens products ............... 115

Richard Vincent Kadison, Strong continuity of operator functions ...

J. G. Kalbfleisch and Ralph Gordon Stanton, Maximal and minimal coverings of $(k-1)$-tuples by $k$-tuples.

Franklin Lowenthal, On generating subgroups of the Moebius group by pairs of infinitesimal transformations...

Michael Barry Marcus, Gaussian processes with stationary increments possessing discontinuous sample paths . .

Zalman Rubinstein, On a problem of Ilyeff ...

Bernard Russo, Unimodular contractions in Hilbert space. ...

David Lee Skoug, Generalized Ilstow and Feynman integrals...

William Charles Waterhouse, Dual groups of vector spaces . 